\title{
Voltage Harmonic Pollution and Treatment Technology of Power Rectifier device
}

\author{
Lixin Zhang ${ }^{1, a}$, Baolin Zhang ${ }^{2, b}$, Changhe $X^{3, c}$ \\ 'Jilin teachers' Institute of Engineering and Technology, Changchun, China \\ ${ }^{2}$ Changchun He Li mechanical and Electrical Equipment Co., Ltd., Changchun, China \\ ${ }^{3}$ Department of equipment of the central reserve grain depot, Changchun, China \\ azlx8015@126.com, bdongnana860901@163.com, ${ }^{\mathrm{c}} 405128987 @ q q . c o m$
}

Keywords: Rectifying device Voltage harmonic Fourier series

Abstract. This article mainly for power uncontrolled rectifier device and controllable rectification device is produced by the voltage harmonic mechanism has carried on the quantitative analysis. This paper illustrates the harm of harmonic of electric power system and harmonic treatment technology.

\section{Introduction}

With the rapid development of power electronic technology and computer control technology, dc power supply, charger, inverter and converter device such as a power converter is widely used in various fields. It brought a lot of convenience for people's production and life. But because of its internal structure have rectifier device. Within the nonlinear components such as diodes, thyristor, insulated gate transistors, etc. These will inevitably produce a large amount of harmonic component. At the same time they give electrical power supply system caused severe harmonic pollution, also for industrial production and human life constitutes the great harm.1 The generation of inverter harmonic.

\section{Uncontrolled rectifier voltage harmonic analysis}

Uncontrolled rectifier device of electricity power is larger. It is generally through the diode three-phase bridge type three-phase alternating current into direct current circuit, the circuit principle as shown in figure 1. Under the condition of impedance load properties, and we don't consider the following LC filter circuit, three-phase bridge rectifier circuit of dc side voltage waveform is shown in figure 2.

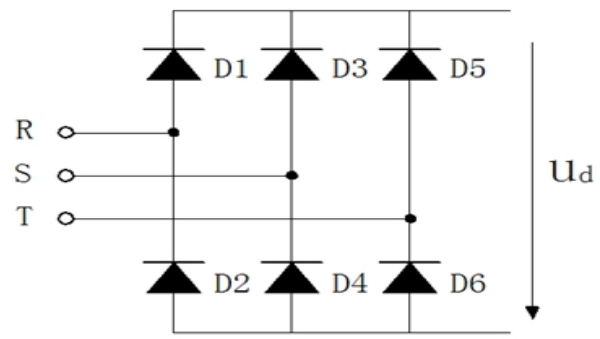

Fig 1. Three-phase bridge rectifier circuit

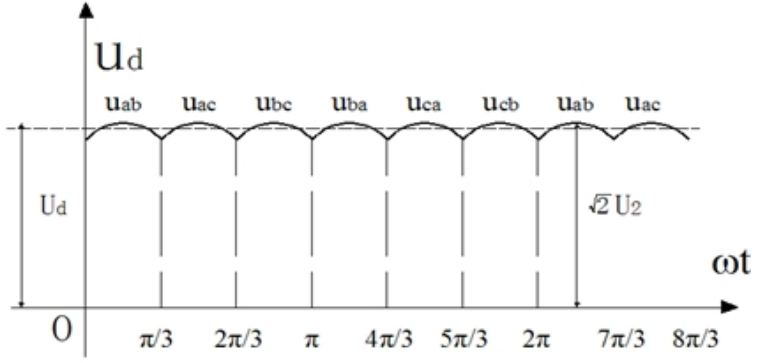

Fig 2. Three-phase bridge rectifier circuit output voltage waveform 


$$
u_{d}=U_{d}\left[1+\sum_{n=m k}^{\infty} C_{n} \cos \left(n \omega t-\varphi_{n}\right)\right] \quad m=6, k=1,2,3 \ldots
$$

Three-phase bridge rectifier output of the circuit for pulsating direct current. It belongs to a non sinusoidal periodic signals. Based on the Fourier series expansion of rectifier output voltage Ud as shown in formula (1). $m$ is rectifying phase number. Three-phase bridge type full-wave rectifier $m$ $=6$.

From the rectifier output voltage expression (1) can draw the conclusion: Rectifier output voltage in addition to the dc component, it also contains a large amount of harmonic component. In order to simplify the analysis and calculation. Move the waveform figure 3 left $\pi / 6$ into standard even functions. The Fourier series expressions coefficient of $b n=0$. The rectifier output voltage expression (1) into type (2).

$$
\begin{aligned}
u_{\mathbf{d}} & =U_{\mathbf{d}}+\sum_{n=m k}^{\infty} a_{n} \cos n \omega t=U_{\mathbf{d}}\left[1-\sum_{n=6 k}^{\infty} \frac{2 \cos k \pi}{n^{2}-1} \cos n \omega t\right]=2.34 U_{2}\left[1-\sum_{n=6 k}^{\infty} \frac{2 \cos k \pi}{n^{2}-1} \cos n \omega t\right] \\
& =2.34 U_{2}\left[1+\frac{2}{35} \cos 6 \omega t-\frac{2}{143} \cos 12 \omega t+\frac{2}{323} \cos 18 \omega t-\frac{2}{575} \cos 24 \omega t+\ldots\right]
\end{aligned}
$$

From formula (2) can be observed, three-phase bridge rectifier output voltage mainly contains the integer harmonic component of the 6 . They are 6, 12, 18, 24 inferior. Their corresponding every harmonic voltage contains at a rate of $5.7 \%, 1.4 \%, 0.6 \%, 0.3 \%$, etc. As every harmonic component of the frequency increased, the amplitude of harmonics in smaller and smaller. Harmonic voltage containing ratio only six times more than the low voltage power grid harmonic standard GB/T14549-1993 even order harmonic of $2 \%$ or less by the rules. So filtering measures must be taken to deal with in reach national standards. Actually after the rectifier circuit connected with LC filter, it can filter out most of the high order harmonic, it focus on 6 times harmonic suppression of dealing with.

\section{Controlled rectifier voltage harmonic analysis}

Electric controlled rectifier devices typically by thyristor three-phase bridge type circuit three-phase alternating current into direct current. The circuit principle as shown in figure 3 . Under the condition of impedance load properties, we do not consider the following LC filter circuit. When triggering Angle $\alpha=\pi / 6$, three-phase bridge controlled rectifier circuit dc side of the output voltage waveform as shown in figure 4.

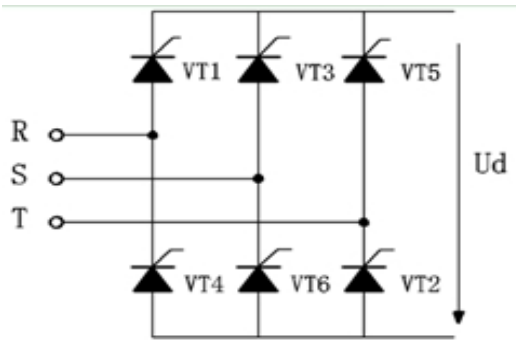

Fig 3. Three-phase bridge controlled rectifier circuit

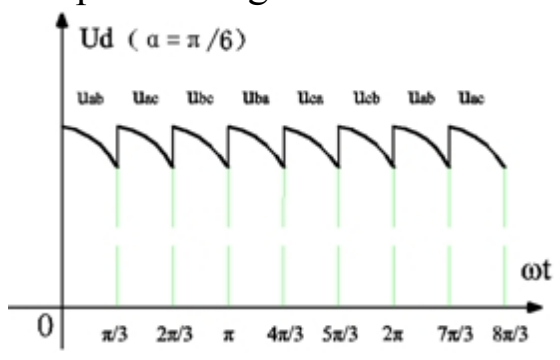

Fig 4. Three-phase bridge controlled rectifier circuit output voltage waveform 
Three-phase controlled bridge rectifier output for pulsating direct current circuit. It belongs to a non sinusoidal periodic signals. Each cycle signal waveform and triggering Angle $\alpha$ values. In $\alpha \leq \pi / 3$ and when the load current is continuous, according to the Fourier series expansion of rectifier output voltage Ud as expression (3). The expression of the calculation is very complicated.

It can be simplified as formula (4) and formula (5).

$$
\begin{aligned}
& u_{\mathbf{d}}=U_{\mathbf{d}}+\sum_{n=1}^{\infty} a_{n} \cos n \omega t+\sum_{n=1}^{\infty} b_{n} \sin n \omega t \quad \mathrm{n}=1,2,3 \ldots \\
& u_{\mathbf{d}}=U_{d}\left[1+\sum_{n=m k}^{\infty} C_{n} \cos \left(n \omega t-\varphi_{n}\right)\right] \quad m=6, \quad \mathrm{k}=1,2,3 \ldots \\
& u_{\mathbf{d}}=2.34 U_{2} \cos \alpha\left[1+\sum_{n=m k}^{\infty} \frac{f_{(n, \alpha)}}{n^{2}-1} \cos \left(n \omega t-\varphi_{n}\right)\right] \quad n=6,12,18
\end{aligned}
$$

From the formula (5) can be observed, the three-phase bridge controlled rectifier output voltage has a large dc component in the Ud. It also contains a lot of integer harmonic component of the six, namely, 6, 12, 18, 24 inferior. It compared with three-phase controlled rectifier circuit, the size of every harmonic component amplitude is not only related to the harmonic number $\mathrm{n}$, but also is closely related to the triggering Angle $\alpha$. When the trigger Angle of $\alpha$ in $0-\pi / 3$ changes, the harmonic component amplitude increased gradually. Thus concluded that the three phase controlled rectifier circuit dc voltage harmonic high 6 multiple times. It more complicated than the uncontrolled rectifier devices harmonic distribution. We must adopt the corresponding harmonic processing technology, avoid it cause great harmonic pollution to power network.

\section{Harm of harmonics}

Voltage harmonic produced by power rectifier device belongs to a kind of electromagnetic interference. It usually by conduction, the electromagnetic radiation and inductive coupling three ways cause harmonic pollution to power grid. So that the grid other user equipment can not work normally or cause electrical accident. The harm of harmonic mainly displays in: lead to public power grid voltage waveform distortion, power transformer and induction motor copper loss and iron loss increased, decreased efficiency, noise, etc.; Power capacitor have even over-voltage over-current, overheating phenomenon; Electric switch and protection equipment insulation damage, tripping misoperation and protection of wrong operation, etc. More should be noted that higher harmonic also for computers, communications equipment, instruments and meters, television remote control equipment, audio equipment and carrier interference, interrupt the communication, and the measured results, use function decline in the quality, etc.

\section{Conclusions}

The disposal method and means a lot of harmonic voltage. General principle is suppression and cut off the harmonic interference sources. Passive filter for general USES capacitance and inductance components. It is in series with the input and output of the rectifier device reactor, thus prevent the public power grid voltage harmonics into communication. Or parallel capacitor voltage harmonic is bypass. Passive filter has the advantage of simple structure, low cost, the disadvantage is that filter out harmonic pertinence is not strong, also can produce line pressure drop, and loss. Active filter is a kind of advanced harmonic treatment technology, it through the detection frequency and amplitude of harmonic voltage rectifier device. Active filter is controlled by a microprocessor automatic filter element to create a harmonic amplitude and offset is equal and opposite polarity current harmonic. It can filter out 2-50 times harmonic component, targeted effect is good, but the price is expensive, so use is limited by a certain range. 


\section{References}

[1] Zhaoan Wang. Power electronic technology.Machinery Industry Press, In Chinese,2005.

[2] Chunhou Tong. Modern AC speed regulation.Metallurgical Industry Press, In Chinese, 1995.

[3] Dazheng Wu. Signal and linear network analysis.Higher Education Press, In Chinese, 2005.

[4] Jianhua Liu. Power electronics and inverter applications.China Labor and social security press, In Chinese, 2009. 